\title{
Ticagrelor or prasugrel vs. clopidogrel in combination with anticoagulation for treatment of acute coronary syndrome in patients with atrial fibrillation
}

\author{
Irene Kirolos, Ikechukwu Ifedili, Miguel Maturana, Alykhan Moez Premji, Brandon Cave, Sherif Roman, \\ David Jones, Romany Gaid, Yehoshua C. Levine, Sunil Jha, Rajesh Kabra, Rami N. Khouzam \\ Department of Medicine, Division of Cardiovascular Diseases, University of Tennessee Health Science Center, Cardiovascular Institute, Methodist \\ Le Bonheur Healthcare System, Memphis TN, USA \\ Contributions: (I) Conception and design: I Kirolos, B Cave, RN Khouzam; (II) Administrative support: I Kirolos; (III) Provision of study materials \\ or patients: I Kirolos; (IV) Collection and assembly of data: I Kirolos, D Jones, I Ifedili, M Maturana, AM Premji, S Roman, R Gaid; (V) Data \\ analysis and interpretation: I Kirolos, D Jones, I Ifedili, M Maturana, AM Premji, S Roman, R Gaid; (VI) Manuscript writing: All authors; (VII) Final \\ approval of manuscript: All authors. \\ Correspondence to: Irene Kirolos, MD. Division of Cardiology, University of Tennessee Health Science Center, Coleman Building, 956 Court Avenue, \\ Memphis, TN 38163, USA. Email: ikirolos@uthsc.edu.
}

\begin{abstract}
For patients with atrial fibrillation (AF) and acute coronary syndrome (ACS), it is often challenging to find the optimal balance between the risk for ischemic and hemorrhagic complication when using both antiplatelet therapy and oral anticoagulation (OAC) with vitamin K antagonist (VKA) or direct oral anticoagulants (DOACs). Current guidelines recommended: (I) double therapy with a P2Y12 inhibitor and dose adjusted VKA is reasonable post-stenting; (II) double therapy with clopidogrel and low-dose rivaroxaban (15 mg daily) may be reasonable post-stenting; (III) double therapy with a P2Y12 inhibitor and dabigatran $150 \mathrm{mg}$ twice daily is reasonable post-stenting. In the AUGUSTUS trial, most patients were given clopidogrel as part a DAPT regimen, however prasugrel and ticagrelor use allowed albeit in a small percentage of the trial population, underestimating its effect. Ticagrelor and prasugrel are known to have a stronger antiplatelet effect compared to clopidogrel, however randomized studies have not been adequately powered to date allowing comparisons between ticagrelor, prasugrel and clopidogrel together in the setting of anticoagulation for the treatment of patients with ACS and AF. Careful consideration should be given to this scenario to avoid falling into the concept of sacrificing efficacy for safety.
\end{abstract}

Keywords: Acute coronary syndrome (ACS); atrial fibrillation (AF); antiplatelets

Submitted Jun 30, 2019. Accepted for publication Jul 12, 2019.

doi: 10.21037/atm.2019.07.41

View this article at: http://dx.doi.org/10.21037/atm.2019.07.41

\section{Introduction}

In the United States, there are more than 5 million patients with atrial fibrillation (AF) (1). Nearly $20 \%$ to $30 \%$ of patients with AF have or will develop coronary artery disease (CAD), leading to more than 100,000 patients annually with AF undergoing percutaneous coronary intervention (PCI) with coronary stenting (2-4). At the same time, nearly $5 \%$ of patients undergoing $\mathrm{PCI}$ already have $\mathrm{AF}$ and require long-term oral anticoagulation (OAC) therapy (5).
Regarding patients with $\mathrm{AF}$ and $\mathrm{CAD}$, it's often challenging to find the optimal balance between the risk for ischemic and hemorrhagic complications in patients requiring both antiplatelet therapy and OAC with a vitamin $\mathrm{K}$ antagonist (VKA) or direct oral anticoagulants (DOACs) (6,7).

The use of dual antiplatelet therapy (DAPT) in combination with an anticoagulant has been referred to as "triple oral antithrombotic therapy". The WOEST trial introduced the concept that removing aspirin (ASA) 
from a triple antithrombotic regimen of ASA, clopidogrel and VKA (e.g., warfarin) may reduce major bleeding without increasing thrombotic risk in patients undergoing PCI and concomitant AF (8). Since, many studies have demonstrated similar findings, the most recent being the AUGUSTUS trial using DOACs (apixaban) (9). Some studies have allowed ticagrelor or prasugrel as an alternative to clopidogrel. We aim to compare the available data with ticagrelor or prasugrel versus clopidogrel in combination with anticoagulation for treatment of patients undergoing $\mathrm{PCI}$ with $\mathrm{AF}$.

\section{Discussion}

Current guidelines for treatment of patients with ACS/AF using dual antiplatelet agents and anticoagulation

American and European guidelines recommend the use of anticoagulation for patients with acute coronary syndrome (ACS) and AF at increased risk of systemic thromboembolism (based on CHA2DS2-VASc risk score), unless the bleeding risk exceeds the expected benefit (10). Current American guidelines, most recently updated in January 2019, Current guidelines recommended the followings:

(I) The use of DAPT with aspirin plus a platelet $\mathrm{P} 2 \mathrm{Y} 12$ inhibitor in addition to warfarin or a nonvitamin K oral anticoagulant ("triple therapy") in patients with AF treated for ACS with a CHA2DS2-VASc score of 2 or greater.

(II) If triple therapy is prescribed for patients with $\mathrm{AF}$ who are at increased risk of stroke and who have undergone PCI with stenting for ACS, a transition to double therapy at 4-6 weeks may be considered.

(III) If triple therapy is prescribed post-stent placement, clopidogrel is preferred over prasugrel.

(IV) Double therapy with a P2Y12 inhibitor and dose adjusted VKA is reasonable post-stenting.

(V) Double therapy with clopidogrel and low-dose rivaroxaban (15 $\mathrm{mg}$ daily) may be reasonable poststenting.

(VI) Double therapy with a P2Y12 inhibitor and dabigatran $150 \mathrm{mg}$ twice daily is reasonable poststenting (11). Based on class IIa recommendation, if triple therapy is prescribed, it is reasonable to choose clopidogrel over prasugrel due to significantly higher risk of bleeding $(11,12)$. Double therapy interventions, combining a P2Y12 inhibitor (clopidogrel or ticagrelor) with an oral anticoagulant (low-dose rivaroxaban, dabigatran or dose-adjusted VKAs) are proposed as alternatives to triple regimen to further reduce the risk of bleeding $(8,13,14)$. Current guidelines suggest an early transition to double therapy may be considered at 4 to 6 weeks during the period of greatest risk of stent thrombosis, if triple therapy was initiated, especially in patients with ST-segment elevation myocardial infarction (MI) $(15,16)$. To date, there are several randomized control trials addressing the topic of anticoagulation after PCI in patients with AF, including the WOEST (8), PIONEER AF-PCI (17) and RE-DUAL PCI (18). A metaanalysis combining these three suggests that the likelihood of an excess of thromboembolic events during dual therapy versus triple therapy is low (19). However, these trials focus on bleeding as the primary endpoint and are underpowered to address ischemic/thromboembolic events including stroke, re-infarction and stent thrombosis (20). The recently released AUGUSTUS trial, and the ongoing ENTRUST-AF-PCI trial (21), will certainly add further information to our current guidelines.

\section{Ticagrelor in PLATO and AUGUSTUS trials}

Based on the PLATO trial results, it was obvious that ticagrelor was superior to clopidogrel in an ACS population (22). Ticagrelor resulted in a $16 \%$ relative risk reduction in death from vascular causes, MI or stroke by compared to clopidogrel. Moreover, there was $21 \%$ significant relative risk reduction in cardiovascular death with ticagrelor versus clopidogrel (22). A benefit of ticagrelor over clopidogrel is not only shown in ACS patients but also for stable CAD patients (23). According to Orme el al. ticagrelor 60 and $90 \mathrm{mg}$ bid regimens had superior platelet inhibition compared toto clopidogrel in stable CAD patients (23).

In the AUGUSTUS trial, among patients with AF treated with long term anticoagulation (apixaban or VKA) undergoing PCI with or without ACS who would require a P2Y12 inhibitor for at least 6 months (9). Apixaban treatment resulted in a statistically significant reduction in major or clinically relevant non-major bleeding and a lower rate of rehospitalization and death compared to VKA therapy (9). The P2Y12 inhibitors included in the trial were clopidogrel, ticagrelor, or prasugrel. However, ticagrelor was only used in $6.2 \%$ of cases despite an ACS population 
of $61.2 \%$. Within this group of patients, there was no significant increase in bleeding risk with either apixaban or VKA use. Ticagrelor had a low percentage use in the trial despite previous trial showing superiority of ticagrelor over clopidogrel in ACS population (22).

\section{Prasugrel use in PIONEER-AF, AUGUSTUS and other trials}

The PIONEER-AF trial, the subgroup analysis of the prasugrel-treated patients was limited to $2 \%$ of $\mathrm{P} 2 \mathrm{Y} 12$ inhibitors prescribed. This small sample size limited its usefulness in subgroup analysis and unable to observe any benefit in terms of bleeding risk, major advanced cardiac events (MACE), time to first stroke, and time to first ischemic stroke (17). Even less use of prasugrel was utilized in the AUGUSTUS trial (9), with only $1.2 \%$ of all patients on treatment and again without any clear conclusions regarding risk or benefit. Despite the lack of data in these trials concerning prasugrel and its risk of bleeding, there are a few single center studies that compared the different P2Y12 inhibitors as part of triple therapy $(11,24)$.

First, Verlinden et al. compared prasugrel/ticagrelor against clopidogrel as part of triple therapy with the primary outcome of incidence of any bleeding during 12-month period after index hospitalization, with secondary outcomes of MACE within 12 months (24). In the 42 patients treated with ticagrelor or prasugrel, $28 \%$ experienced a bleeding event, compared to $12.7 \%$ of patients treated with clopidogrel (OR 3.3; 95\% CI, 1.38-8.34; $\mathrm{P}=0.017$ ). There was no significant difference between the groups for the outcomes of MACE, MI, ischemic stroke, or cardiac death.

Second, Sarafoff et al. looked at 377 patients who underwent successful stenting and platelet function testing and were discharged with a 6 -month regimen of triple anticoagulation therapy (11). Among these patients 21 were treated with prasugrel of which 18 were due to high platelet reactivity despite clopidogrel treatment. Even though the study was not powered to assess clinical endpoints, including thrombolysis in MI (TIMI) major and minor bleeding at 6 months. Combined major and minor bleeding was seen in $28.6 \%$ of patients given prasugrel, compared to $6.7 \%$ of those given clopidogrel (HR 4.6; 95\% CI, $1.9-11.4 ; \mathrm{P}<0.001)$. There was no significant difference in MACE. However, in both of these studies warfarin was the oral anticoagulant used in the study in conjunction with ASA. While these data are from single centers, and have small sample sizes, they underline the need for more careful consideration of prasugrel as a component of triple therapy.

\section{Oral anticoagulant in PIONEER-AF and AUGUSTUS trials}

The PIONEER-AF trial sought to understand if there was a difference in the risk of bleeding for patients with nonvalvular AF undergoing PCI with stent placement. Low dose rivaroxaban with single or DAPT reduced the risk of TIMI, major and minor bleeding when compared to warfarin with DAPT at 1 year.

The AUGUSTUS trial sought to understand whether OAC with apixaban is superior to warfarin with regard to thrombotic events and bleeding, and whether the addition of ASA to OAC with P2Y12 inhibitor therapy is superior to placebo. The study found that apixaban is associated with a $4.2 \%$ absolute reduction in major bleeding compared to warfarin, and ASA use in addition to OAC and P2Y12 inhibitor therapy was associated with a $7.1 \%$ absolute increase in major bleeding. There was no difference in thrombotic events or for secondary outcomes.

In summary, in the group of patients where antiplatelet and anticoagulation therapy is needed, the preference between ticagrelor, prasugrel and clopidogrel was difficult to decide upon, and this is due to many factors. First, most of the leading studies focus on bleeding as primary outcome and not thrombotic outcomes, which is the desired outcome when choosing more potent P2Y12 inhibitors $(19,20)$. Consequently, it is difficult to compare different antiplatelets agents' effect on thrombotic outcome when used together with anticoagulation.

Second, in case of ticagrelor for example, previous studies done comparing clopidogrel to ticagrelor, already proved that ticagrelor is more efficient and superior to clopidogrel for prevention of thrombotic events (22), therefore, there is a considerable chance that ticagrelor will be more efficient than clopidogrel in combination with anticoagulation in preventing thrombotic events. Therefore, we should explore more in that regard and not to fall in the pit of sacrificing efficacy for safety.

Third, Verlinden trial (24) and Sarafoff et al. (11) were underpowered to assess clinical endpoints, including TIMI major and minor bleeding at 6 months, therefore, well powered studies are needed. Moreover, in both of these studies, only warfarin was used. Caldeira et al. (25) showed that DOACs were at least as effective as VKAs in preventing stroke in AF patients and were associated with less bleeding, particularly less ICH, which is intriguing as DOACs may offset the increased bleed risk of potent antiplatelet therapy. 
Additionally, both Verlinden et al. and Sarafoff et al. included ASA and did not evaluate dual antithrombotic therapy.

Fourth, the AUGUSTUS trial, which is the most recent randomized trial for the use of antiplatelet and anticoagulant together, was not powered sufficiently to compare outcomes between different antiplatelet agents. More randomized trials need to be conducted for further clarification of the choice of antiplatelet in combination with anticoagulation in this challenging group of patients diagnosed with ACS and AF.

\section{Conclusions}

Clopidogrel is the current default P2Y12 inhibitor used in ACS/AF patients recommended by guidelines. Ticagrelor and prasugrel are known to have a stronger antiplatelet effect yet are not used because of the increased risk whether real or perceived which have not been confirmed with large randomized studies in this patient population. In AUGUSTUS, only 9 patients needed to be treated to prevent a major or clinically-relevant non-major bleeding event and only 18 patients needed to be treated to prevent a death or hospitalization when comparing apixaban plus a P2Y12 inhibitor to triple therapy with VKA, P2Y12 inhibitor plus aspirin. With the robust safety benefit of DOACs, perhaps the most intriguing combination may be with the ticagrelor or prasugrel to minimize the increased bleeding risk in combination. However, well-designed studies are justified to better inform choice of P2Y12 inhibitor used until this strategy can be widely implemented

\section{Acknowledgments}

None.

\section{Footnote}

Conflicts of Interest: The authors have no conflicts of interest to declare.

Ethical Statement: The authors are accountable for all aspects of the work in ensuring that questions related to the accuracy or integrity of any part of the work are appropriately investigated and resolved.

\section{References}

1. Miyasaka Y, Barnes ME, Gersh BJ, et al. Secular
Trends in Incidence of Atrial Fibrillation in Olmsted County, Minnesota, 1980 to 2000, and Implications on the Projections for Future Prevalence. Circulation 2006;114:119-25.

2. Moser M, Olivier CB, Bode C. Triple antithrombotic therapy in cardiac patients: more questions than answers. Eur Heart J 2014;35:216-23.

3. Dewilde WJ, Janssen PW, Verheught FW, et al. Triple therapy for atrial fibrillation and percutaneous coronary intervention. J Am Coll Cardiol 2014;64:1270-80.

4. Jolly SS, Natarajan MK. Atrial fibrillation and PCI-do we still need aspirin? N Engl J Med 2016;375:2490-2.

5. Lip GY, Windecker S, Huber K, et al. Management of antithrombotic therapy in atrial fibrillation patients presenting with acute coronary syndrome and/or undergoing percutaneous coronary or valve interventions: a joint consensus document of the European Society of Cardiology Working Group on Thrombosis, European Heart Rhythm Association (EHRA), European Association of Percutaneous Cardiovascular Interventions (EAPCI) and European Association of Acute Cardiac Care (ACCA) endorsed by the Heart Rhythm Society (HRS) and AsiaPacific Heart Rhythm Society (APHRS). Eur Heart J 2014:35:3155-79.

6. Alexopoulos D, Vlachakis P, Lekakis J. Triple antithrombotic therapy in atrial fibrillation patients undergoing PCI: a fading role. Cardiovasc Drugs Ther 2017;31:319-24.

7. Liu J, Fan M, Zhao J, et al. Efficacy and safety of antithrombotic regimens after coronary intervention in patients on oral anticoagulation: traditional and Bayesian metaanalysis of clinical trials. Int J Cardiol 2016;205:89-96.

8. Dewilde WJ, Oirbans T, Verheugt FW, et al. Use of Clopidogrel with or without aspirin in patients taking oral anticoagulant therapy and undergoing percutaneous coronary intervention: an open-label, randomised, controlled trial. Lancet 2013;381:1107-15.

9. Lopes RD, Heizer G, Aronson R, et al. Antithrombotic Therapy after Acute Coronary Syndrome or PCI in Atrial Fibrillation. N Engl J Med 2019;380:1509-24.

10. January CT, Wann LS, Calkins H, et al. 2019 AHA/ ACC/HRS Focused Update of the 2014 AHA/ACC/ HRS Guideline for the Management of Patients With Atrial Fibrillation: A Report of the American College of Cardiology/American Heart Association Task Force on Clinical Practice Guidelines and the Heart Rhythm Society in Collaboration With the Society of Thoracic Surgeons. Circulation 2019;140:e125-51. 
11. Sarafoff N, Martischnig A, Wealer J, et al. Triple therapy with aspirin, Prasugrel, and vitamin $\mathrm{K}$ antagonists in patients with drug-eluting stent implantation and an indication for oral anticoagulation. J Am Coll Cardiol 2013;61:2060-6.

12. Jackson LR 2nd, Ju C, Zettler M, et al. Outcomes of patients with acute myocardial infarction undergoing percutaneous coronary intervention receiving an oral anticoagulant and dual antiplatelet therapy: a comparison of Clopidogrel versus Prasugrel from the TRANSLATEACS Study. JACC Cardiovasc Interv 2015;8:1880-9.

13. Lamberts M, Gislason GH, Olesen JB, et al. Oral anticoagulation and antiplatelets in atrial fibrillation patients after myocardial infarction and coronary intervention. J Am Coll Cardiol 2013;62:981-9.

14. Braun OÖ, Bico B, Chaudhry U, et al. Concomitant use of warfarin and Ticagrelor as an alternative to triple antithrombotic therapy after an acute coronary syndrome. Thromb Res 2015;135:26-30.

15. Fiedler KA, Maeng M, Mehilli J, et al. Duration of triple therapy in patients requiring oral anticoagulation after drug-eluting stent implantation: the ISAR-TRIPLE Trial. J Am Coll Cardiol 2015;65:1619-29.

16. Koskinas KC, Räber L, Zanchin T, et al. Duration of triple antithrombotic therapy and outcomes among patients undergoing percutaneous coronary intervention. JACC Cardiovasc Interv 2016;9:1473-83.

17. Gibson CM, Mehran R, Bode C, et al. Prevention of Bleeding in Patients with Atrial Fibrillation Undergoing PCI. N Engl J Med 2016;375:2423-34.

18. Cannon CP, Bhatt DL, Oldgren J, et al. Dual Antithrombotic Therapy with Dabigatran after PCI in

Cite this article as: Kirolos I, Ifedili I, Maturana M, Premji AM, Cave B, Roman S, Jones D, Gaid R, Levine YC, Jha S, Kabra R, Khouzam RN. Ticagrelor or prasugrel vs. clopidogrel in combination with anticoagulation for treatment of acute coronary syndrome in patients with atrial fibrillation. Ann Transl Med 2019;7(17):406. doi: 10.21037/atm.2019.07.41
Atrial Fibrillation. N Engl J Med 2017;377:1513-24.

19. Piccini JP, Jones WS. Triple therapy for atrial fibrillation after PCI. N Engl J Med 2017;377:1580-2.

20. Steffel J, Verhamme P, Potpara TS, et al. The 2018 European Heart Rhythm Association Practical Guide on the use of non-vitamin $\mathrm{K}$ antagonist oral anticoagulants in patients with atrial fibrillation. Eur Heart J 2018;39:1330-93.

21. Vranckx P, Lewalter T, Valgimigli M, et al. Evaluation of the safety and efficacy of an edoxaban-based antithrombotic regimen in patients with atrial fibrillation following successful percutaneous coronary intervention (PCI) with stent placement: Rationale and design of the ENTRUST-AF PCI trial. Am Heart J 2018;196:105-12.

22. Wallentin L, Becker RC, Budaj A, et al. Ticagrelor versus Clopidogrel in patients with acute coronary syndromes. N Engl J Med 2009;361:1045-57.

23. Orme RC, Parker WAE, Thomas MR, et al. Study of Two Dose Regimens of Ticagrelor Compared with Clopidogrel in Patients Undergoing Percutaneous Coronary Intervention for Stable Coronary Artery Disease (STEELPCI). Circulation 2018. [Epub ahead of print].

24. Verlinden NJ, Coons JC, Iasella CJ, et al. Triple Antithrombotic Therapy With Aspirin, P2Y12 Inhibitor, and Warfarin After Percutaneous Coronary Intervention: An Evaluation of Prasugrel or Ticagrelor Versus Clopidogrel. J Cardiovasc Pharmacol Ther 2017;22:546-51.

25. Caldeira D, Rodrigues FB, Barra M, et al. Non-vitamin K antagonist oral anticoagulants and major bleeding-related fatality in patients with atrial fibrillation and venous thromboembolism: a systematic review and meta-analysis. Heart 2015;101:1204-11. 